\title{
Elastic Modes and Their Computation
}

\author{
G.W. Hodstrom
}

April 1995

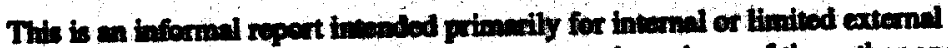
distribution. The opiaions and conclusions steted ere those of the suthor and may or may not be those of the Loboracory.

Work performed under the enspices of the U.S. Depurtment of Energy by the Lawrence Livermore National Labontory under Contract W-7405-ENG-48. 


\section{DLCLAMER}

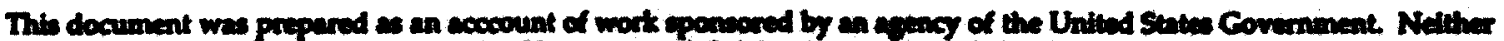

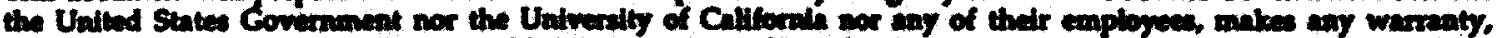

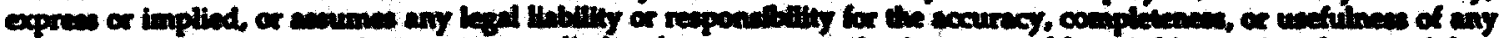

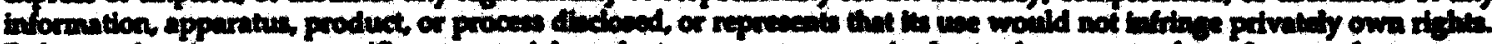

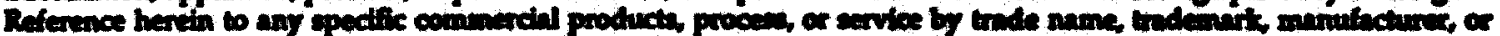

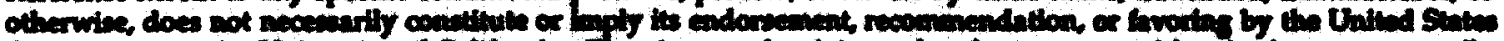

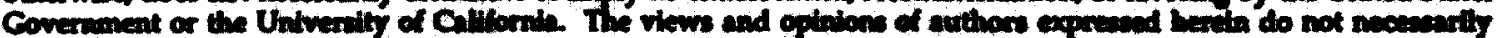

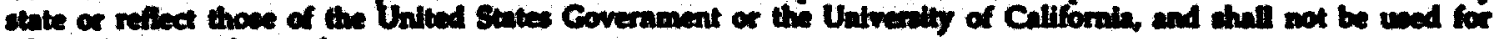
advertung or prodect endorseneat purposen.

Tis report hes bean repoduced directly trom the bet avillate copy.

Avalioble to DOS and DOB contractese from the Ofice of Sclentise and Techaicl belomation

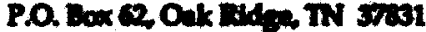

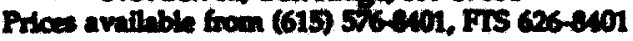

Avalible to the public from the

National Technical Infermation Service

US. Depertmant of Commerce 52.5 Pont Roynd Rd. Springfield, VA 22161 


\title{
Elastic modes and their computation
}

\author{
G. W. Hedstrom \\ Lawrence Livermore National Laboratory \\ P. O. Box 808, L-321 \\ Livermore, CA 94551
}

\begin{abstract}
In this note we summarize the theory of modes in stratified elastic media, and we discuss some of the considerations necessary to achieve reliable numerical computations. We also point out the consequences of the fact that the corresponding eigenvalue problem is not selfadjoint.
\end{abstract}

1. Introduction. We begin with a discussion of the theory of modes for stratified isotropic linear elastic media, including the coupling to liquid layers. We then take up the implications of these ideas for numerical computation. These are topics which have been treated by numerous previous authors, including Aki and Richards [1, pp. 267-286], Kennett [7, Ch. 2], and Porter and Reiss [8], but we emphasize the computational considerations.

We work in cylindrical coordinates $(r, z, \theta)$. With the notation that $u^{T}$ denotes the transpose of $u$, the unknowns in cylindrical coordinates are the displacement

$$
u=\left(u_{r}, u_{z}, u_{\theta}\right)^{T}
$$

and the stress tensor

$$
\tau=\left(\begin{array}{lll}
\tau_{r r} & \tau_{\tau z} & \tau_{r \theta} \\
\tau_{r z} & \tau_{z z} & \tau_{z \theta} \\
\tau_{r \theta} & \tau_{z \theta} & \tau_{\theta \theta}
\end{array}\right) .
$$

The domain of interest is the halfspace $z>0$, with a free surface at $z=0$.

With no body forces Newton's second law gives $[6, p .213]$

$$
\begin{aligned}
& \rho \partial_{t}^{2} u_{r}=\partial_{r} \tau_{r r}+\partial_{z} \tau_{r z}+\frac{1}{r} \partial_{\theta} \tau_{r \theta}+\frac{1}{r}\left(\tau_{r r}-\tau_{\theta \theta}\right), \\
& \rho \partial_{t}^{2} u_{z}=\partial_{r} \tau_{r z}+\partial_{z} \tau_{z z}+\frac{1}{r} \partial_{\theta} \tau_{z \theta}+\frac{1}{r} \tau_{r z}, \\
& \rho \partial_{t}^{2} u_{\theta}=\partial_{r} \tau_{r \theta}+\partial_{z} \tau_{z \theta}+\frac{1}{r} \partial_{\theta} \tau_{\theta \theta}+\frac{2}{r} \tau_{r \theta} .
\end{aligned}
$$

where $\rho$ denotes the density of the material. In terms of the Lamé parameters $\lambda$ and $\mu$ the constitutive relation for a linear isotropic medium takes the form $[6$, 
p. 212-213]

$$
\begin{aligned}
& \tau_{r r}=\lambda P+2 \mu \partial_{r} u_{r}, \\
& \tau_{z z}=\lambda P+2 \mu \partial_{z} u_{z}, \\
& \tau_{\theta \theta}=\lambda P+\frac{2 \mu}{r}\left(\partial_{\theta} u_{\theta}+u_{r}\right), \\
& \tau_{r z}=\mu\left(\partial_{r} u_{z}+\partial_{z} u_{r}\right), \\
& \tau_{r \theta}=\mu\left(\partial_{r} u_{\theta}-\frac{u_{\theta}}{r}+\frac{\partial_{\theta} u_{r}}{r}\right), \\
& \tau_{z \theta}=\mu\left(\partial_{z} u_{\theta}+\frac{\partial_{\theta} u_{z}}{r}\right)
\end{aligned}
$$

with

$$
P=\partial_{r} u_{r}+\partial_{z} u_{z}+\frac{1}{r}\left(\partial_{\theta} u_{\theta}+u_{r}\right)
$$

For the system (1.3-1.5) we make the simplifying assumption that the material is horizon tally stratified, so that $\rho, \lambda$, and $\mu$ depend only on the depth $z$. Consequently, we may represent the solution of (1.3-1.5) in terms of a Fourier series in $\theta$, a Fourier transform with respect to time, and a Hankel transform with respect to $r$. The $\theta$-dependence $\exp \{i m \theta\}$ of the solution is determined by the initial conditions as follows. For an axially-symmetric source we have $m=0$, for a vertical double couple we have $m= \pm 1$, and for a horizontal double couple we have $m= \pm 2$.

It is well known (cf., [1, pp. 267-286] and [7, Ch. 2]) that after the transformations mentioned above, the horizontally-polarized shear waves ( $S H$-waves) may be separated from the compressional and vertically-polarized shear waves $(P-S V$ waves). In Section 2 we write each of these systems in the form

$$
\partial_{z} \Phi-A \Phi=k^{2} B \Phi,
$$

where $\Phi$ is a vector derived from the Fourier-Hankel transforms of components of $u$ and $\tau$. In (1.6) $A$ and $B$ are matrices depending on the frequency $\omega$, the density $\rho$, the Lamé parameters $\lambda$ and $\mu$, and the depth $z$. In Section 6 we show that acoustic waves in water also satisfy an equation of the form (1.6).

The equation (1.6) looks like an eigenvalue problem with eigenvalue $k^{2}$. Seemingly, one needs only to prescribe boundary conditions as follows. The traction $\left(\tau_{r r}, \tau_{r z}, \tau_{z \theta}\right)$ vanishes at the free surface, $z=0$. If the problem has an interface between water and solid, then at that surface $u_{z}$ and $\tau_{z z}$ are continuous, and the shear stress components $\tau_{r z}$ and $\tau_{z \theta}$ vanish. The final boundary condition is that there are no incoming waves from infinite depth.

The system (1.6) is not in standard Sturm-Liouville form for an eigenvalue problem for two reasons. For one thing, the matrices $A$ and $B$ are nonsymmetric. More 
important, the matrix $B$ has determinant zero. For the $S H$-system and the system for acoustic waves in water there are transformations taking (1.6) to Sturm-Liouville form, but no such transformation exists for the $P-S V$-system. The consequences of this situation were discussed by Chapman and Woodhouse [3] and Woodhouse [14], and they are examined in more detail in Section 3. The most significant of these is that one must also solve the adjoint equation

$$
-\partial_{z} \Psi^{T}-\Psi^{T} A=k^{2} \Psi^{T} B
$$

Observe that the adjoint equation (1.7) involves the transposition operation $T$, but it does not involve complex conjugation. The boundary conditions for (1.7) are chosen so as to obtain the following biorthogonality relation. Suppose that we have two eigenvalues $k_{n}$ and $k_{\ell}$ with $k_{n}^{2} \neq k_{\ell}^{2}$. Suppose also that $\Phi(n)$ is a solution of (1.6) with $k=k_{n}$ and that $\Psi(\ell)$ satisfies (1.7) with $k=k_{\ell}$, each with appropriate boundary conditions. Then the biorthogonality relation is

$$
\int_{0}^{\infty} \Psi(\ell)^{T} B \Phi(n) d z=0
$$

The biorthogonality is important in that it determines the form taken by the equation for the coefficients of the eigenfunction expansion.

Section 4 begins our discussion of computational considerations. We have chosen to use the simplest approximation of Preuss type [9], in which the matrices $A$ and $B$ are replaced by piecewise-constant matrices. That is, we replace the medium by homogeneous layers. One may use a more accurate method, such as by using layers in which the $P$-wave speed is linear and the $S$-wave speed constant [2]. The issues we treat here extend immediately to such methods.

The approximation of the medium by homogeneous layers converts the eigenvalue problem (1.6) to one of finding the zeros of the determinant of a matrix. Therefore in Section 5 we examine the question of computing such determinants in a numerically stable manner. Finally, Section 6 is devoted to the modifications introduced when one of the layers is a fluid.

2. $S H$ - and $P$-SV-waves. In this section we identify the vector $\Phi$ and the matrices $A$ and $B$ corresponding to the eigenvalue problem (1.6) for horizontallypolarized shear waves ( $\mathrm{SH}$-waves) and the system of compressional waves and vertically-polarized shear waves ( $P-S V$-waves) in a linear, isotropic, horizontally-stratified elastic medium. Our presentation follows that of Woodhouse [13] and Takeuchi and Saito [11].

Fourier-Hankel representation for $P-S V$-waves. Let $m$ be an integer and let $J_{m}$ be the corresponding Bessel function. For modes with angular and radial dependence

$$
Y_{k}^{m}(r, \theta)=J_{m}(k r) \exp \{i m \theta\}
$$


the $P-S V$-waves are polarized in the vertical tangent plane containing the horizontal vector $\nabla Y_{k}^{m}$. Let $e_{r}, e_{z}$, and $e_{\theta}$ denote, respectively, the unit vectors in the $r, z$, and $\theta$ directions. Then for $P-S V$-waves the components of the vector $\Phi$ in (1.6) corresponding to the displacement are

$$
\begin{aligned}
u_{\tau} e_{r}+u_{\theta} e_{\theta} & =\frac{\Phi_{1}(z)}{k} \nabla Y_{k}^{m}(r, \theta) \exp \{i \omega t\} \\
u_{z} e_{z} & =k \Phi_{2}(z) Y_{k}^{m}(r, \theta) \exp \{i \omega t\} e_{z} .
\end{aligned}
$$

The remaining components of $\Phi$ are related to the traction $\tau \cdot e_{z}$ on a horizontal plane according to the relations

$$
\begin{aligned}
\tau_{r z} e_{r}+\tau_{z \theta} e_{\theta} & =\frac{\Phi_{3}(z)}{k} \nabla Y_{k}^{m}(r, \theta) \exp \{i \omega t\} \\
\tau_{z z} e_{z} & =k \Phi_{4}(z) Y_{k}^{m}(r, \theta) \exp \{i \omega t\} e_{z} .
\end{aligned}
$$

The $P-S V$ eigenvalue problem. Now that we have selected a vector $\Phi$ for cylindrical $P-S V$-wave solutions of (1.3-1.5), we write the corresponding matrices $A$ and $B$ for the $P-S V$-system (1.6). We refer the reader to Woodhouse [13], where it is shown that for $P-S V$-waves with $\Phi$ as in (2.2-2.3) we have

$$
A=\left(\begin{array}{cccc}
0 & -1 & 1 / \mu & 0 \\
0 & 0 & 0 & 1 /(\lambda+2 \mu) \\
-\rho \omega^{2} & 0 & 0 & -\lambda /(\lambda+2 \mu) \\
0 & -\rho \omega^{2} & 0 & 0
\end{array}\right)
$$

and

$$
B=\left(\begin{array}{cccc}
0 & 0 & 0 & 0 \\
\lambda /(\lambda+2 \mu) & 0 & 0 & 0 \\
4 \mu(\lambda+\mu) /(\lambda+2 \mu) & 0 & 0 & 0 \\
0 & 0 & 1 & 0
\end{array}\right)
$$

Observe that with $A$ and $B$ as in (2.4-2.5) equation (1.6) is very much a nonstandard eigenvalue problem because the rank of the matrix $B$ in (2.5) is only 2 .

Boundary conditions for $P$-SV-waves. For an elastic halfspace $0<z<\infty$ with a free surface at $z=0$ one boundary condition for (1.6) is that the traction $\tau \cdot e_{z}$ on the free surface vanish,

$$
\Phi_{3}=0 \text { and } \Phi_{4}=0 \text { at } z=0 .
$$

On physical grounds the other boundary condition is,

$$
\text { no incoming waves from } z=\infty \text {. }
$$

Note that because inhomogeneities in a material induce backscattering of waves, the condition (2.7) makes sense only if the material properties are constant for 
large values of $z$. That is, we assume the presence of a foundation consisting of a homogeneous halfspace. The precise meaning of the condition (2.7) at infinity for a homogeneous halfspace will be made clear in Section 4.

We are also interested in the boundary conditions for a solid-fluid interface, but we postpone a discussion until we treat fluid layers in Section 6.

The $S H$ eigenvalue problem. For $S H$-waves we again use the cylindrical functions $Y_{k}^{m}$ defined in (2.1). For the displacement component $\Phi_{1}$ in (1.6) we follow Woodhouse [13] in using

$$
u_{r} e_{r}+u_{\theta} e_{\theta}=\frac{\Phi_{1}(z)}{k} e_{z} \times \nabla Y_{k}^{m}(r, \theta) \exp \{i \omega t\}
$$

Furthermore, the stress components of an $S H$-wave are given by

$$
\tau_{r z} e_{r}+\tau_{z \theta} e_{\theta}=\frac{\Phi_{2}(z)}{k} e_{z} \times \nabla Y_{k}^{m}(r, \theta) \exp \{i \omega t\}
$$

A substitution of (2.8-2.9) into (1.3-1.5) gives the equation (1.6) with the matrices $A$ and $B$ given by

$$
A=\left(\begin{array}{cc}
0 & 1 / \mu \\
-\rho \omega^{2} & 0
\end{array}\right)
$$

and

$$
B=\left(\begin{array}{ll}
0 & 0 \\
\mu & 0
\end{array}\right)
$$

The boundary conditions are that $\Phi_{2}=0$ at a free surface or at a solid-fluid interface and that there be no incoming waves from infinite depth.

It is clear that the matrix $B$ in (2.11) is rank deficient, but in the case of (2.102.11) there exists a transformation of (1.6) to Sturm-Lioville form. In fact, upon elimination of $\Phi_{2}$, we find that $\Phi_{1}$ satisfies the selfadjoint equation

$$
\partial_{x}\left(\mu \partial_{x} \Phi_{1}\right)+\rho \omega^{2} \Phi_{1}=k^{2} \mu \Phi_{1}
$$

One way to view this situation is to say that for $S H$-waves the displacement may be expanded in terms of eigenfunctions, but the shear stress is a derived quantity to be obtained from the displacement by (2.9) and

$$
\Phi_{2}=\mu \partial_{2} \Phi_{1}
$$

The only possible peculiarity we might mention about (2.12) is that there is no inherent reason why $k^{2}$ must be positive. Thus, it is perfectly reasonable that $k$ in (2.12) be pure imaginary. Such points in the spectrum represent waves which propagate vertically and (by (2.8)) decay radially. We shall find in Section 4 that we must also include the possibility of $P-S V$-waves of this sort. 
3. The adjoint $P-S V$ problem. In this section we examine the adjoint equation (1.7) in the $P-S V$ case. That is, the matrices $A$ and $B$ are given by (2.4-2.5). We present the result of Chapman and Woodhouse [3] showing that the adjoint eigenfunction $\Psi$ may be written in terms of a permutation and scaling of the components of $\Phi$. We then discuss the implications of this result for eigenfunction expansions.

The boundary conditions for (1.7) are chosen so as to ensure that for every solution $\Phi$ of (1.6) with boundary conditions (2.6-2.7) the boundary terms vanish in the formula for integration by parts,

$$
\int_{0}^{\infty} \Psi^{T} \partial_{z} \Phi d z=-\int_{0}^{\infty}\left(\partial_{z} \Psi^{T}\right) \Phi d z
$$

That is, we want

$$
\sum_{j=1}^{4} \Psi_{j} \Phi_{j}=0
$$

at $z=0$ and $z=\infty$. Because of (2.6), we may obtain (3.2) at $z=0$ by requiring

$$
\Psi_{1}=0 \text { and } \Psi_{2}=0 \text { at } z=0 .
$$

The condition at infinity requires further discussion, and in Section 5 it will be shown to be equivalent to

$\Psi$ has no incoming waves at $z=\infty$.

Representation of $\Psi$ in terms of $\Phi$. We now show that the adjoint eigenfunction $\Psi$ may be written as a permutation and scaling of the components of $\Phi$, specifically, $\Psi=Q \Phi$ with $Q$ given by

$$
Q=\left(\begin{array}{cccc}
0 & 0 & -k^{2} & 0 \\
0 & 0 & 0 & -1 \\
k^{2} & 0 & 0 & 0 \\
0 & 1 & 0 & 0
\end{array}\right)
$$

Theorem 3.1 (Chapman and Woodhouse [3]). Let $Q$ denote the matrix (3.5). If $\Phi$ is a solution of the equation (1.6) with $A$ and $B$ as given by (2.4-2.5) with boundary conditions (2.6-2.7), then $\Psi=Q \Phi$ is a solution of the adjoint problem (1.7) with boundary conditions (3.3-3.4).

Proof. We start by computing $\Phi=Q \Psi$,

$$
\Psi=Q \Phi=\left(\begin{array}{llll}
-k^{2} \Phi_{3}, & -\Phi_{4}, & k^{2} \Phi_{1}, & \Phi_{2}
\end{array}\right)^{T} .
$$

It then follows by direct substitution that $\Psi$ is a solution of

$$
-\partial_{z} \Psi=\left(A^{T}+k^{2} B^{T}\right) \Psi
$$


if $\Phi$ satisfies (1.6) with $A$ and $B$ as given by (2.4-2.5). This proves that $\Psi$ satisfies the adjoint equation (1.7).

It remains to verify that $\Psi=Q \Phi$ satisfies the boundary conditions (3.3-3.4). Because of (2.6), it is clear from (3.6) that the conditions (3.3) at $z=0$ are satisfied. We postpone until the next section a proof of the fact that for a homogeneous halfspace the outgoing condition at $z=\infty$ for $\Phi$ in $(2.7)$ is carried over to $\Psi$ by (3.6). Once this is done, the theorem will be proved.

Remarks. The reader may be familiar with applications in which the solution of the adjoint problem involves the complex conjugate of the eigenfunction. This does not happen in our case because the matrix $A+k^{2} B$ is not skew hermitian. Note also that any nonzero constant multiple of $Q \Phi$ is a solution of the adjoint problem (1.7) with boundary conditions (3.3-3.4). The normalization is a matter of convenience.

Orthogonality relations. We assume that the boundary-value problem (1.6) in the $P-S V$-case (2.4-2.7) has some discrete eigenvalues $k_{\ell}$, counted by integer $\ell$. Let $\Phi(\ell)$ and $\Psi(\ell)^{T}$ denote the corresponding eigenfunctions. That is, $\Phi(\ell)$ is not identically zero and satisfies differential equation (1.6) with $k=k_{\ell}$ and with boundary conditions (2.6-2.7). Then $\Psi(\ell)=Q \Phi(\ell)$ is a nontrivial solution of the adjoint problem (1.7) with $k=k_{\ell}$. If $k_{\ell}$ and $k_{n}$ are two different eigenvalues, then the orthogonality relation is that

$$
\int_{0}^{\infty} \Psi(\ell)^{T} B \Phi(n) d z=0
$$

This is proved by first writing (1.6) for $k_{n}$ and $\Phi(n)$, multiplying on the left by $\Psi(\ell)^{T}$, and integrating to get

$$
k_{n}^{2} \int_{0}^{\infty} \Psi(\ell)^{T} B \Phi(n) d z=\int_{0}^{\infty}\left(\Psi(\ell)^{T} \partial_{z} \Phi(n)-\Psi(\ell)^{T} \cdot A \Phi(n)\right) d z
$$

We then apply integration by parts (3.1) to the first term on the right-hand side of (3.9) and use the adjoint equation (1.7) to show that

$$
k_{n}^{2} \int_{0}^{\infty} \Psi(\ell)^{T} B \Phi(n) d z=k_{\ell}^{2} \int_{0}^{\infty} \Psi(\ell)^{T} B \Phi(n) d z
$$

The assumption $k_{n}^{2} \neq k_{\ell}^{2}$ now implies the orthogonality relation (3.8).

Eigenfunction expansions. The orthogonality relation (3.8) together with $\Psi(\ell)=Q \Phi(\ell)$ suggests the 'inner product'

$$
\langle\Phi(\ell), \Omega\rangle=\int_{0}^{\infty} \Phi(\ell)^{T} Q^{T} B \Omega d z
$$


Upon expansion of the integrand, we obtain

$$
\langle\Phi(\ell), \Omega\rangle=\frac{1}{\lambda+2 \mu} \int_{0}^{\infty}\left(4 k^{2} \mu(\lambda+\mu) \Phi(\ell)_{1} \Omega_{1}+(\lambda+2 \mu) \Phi(\ell)_{2} \Omega_{3}-\lambda \Phi(\ell)_{4} \Omega_{1}\right) d z .
$$

This is a very unusual 'inner product', and we use quotes because we do not have commutativity, $\langle\Omega, \Phi(\ell)\rangle \neq\langle\Phi(\ell), \Omega\rangle$. Furthermore, we write the 'inner product' as $\langle\Phi(\ell), \Omega\rangle$ in order to emphasize the fact that in applications the first factor is always an eigenfunction. In spite of these difficulties, let us proceed formally. By writing (3.8) in the form

$$
\langle\Phi(\ell), \Phi(n)\rangle=0 \text { for } \ell \neq n,
$$

it becomes easy to obtain a formula for the expansion coefficients $c(n)$ in

$$
\Omega=\sum_{n} c(n) \Phi(n)
$$

In fact, upon taking the 'inner product' of $\Phi(\ell)$ with (3.11) term by term, we find that

$$
c(\ell)=\frac{\langle\Phi(\ell), \Omega\rangle}{\langle\Phi(\ell), \Phi(\ell)\rangle}
$$

Remarks. The denominator of (3.12) suggests the normalization $\langle\Phi(\ell), \Phi(\ell)\rangle=$ 1 , but from (3.10) it is not clear that $(\Phi(\ell), \Phi(\ell)\rangle$ is positive, or even that it is nonzero. In our computations we have not seen any cases of $\langle\Phi(\ell), \Phi(\ell)\rangle \leq 0$, however. If it should happen that $\langle\Phi(\ell), \Phi(\ell)\rangle=0$ for some value of $\ell$, then an expansion (3.11) is impossible unless $\langle\Phi(\ell), \Omega\rangle=0$, and in that case $c(\ell)$ is indeterminate.

Note that in our use of the 'inner product' in (3.12) the factor $\Phi(\ell)$ is an eigenfunction. Recall also that in our discussion of the eigenvalue problem for $S H$-waves, we used the differential equation (2.13) to eliminate one component in order to obtain the equation for the other component (2.12) in standard form. These considerations suggest that we might be able to a more reasonable 'inner product' by making analogous substitutions in (3.10). In fact, because the components $\Omega_{2}$ and $\Omega_{4}$ play no rôle in the product $B \Omega$, it seems natural to eliminate $\Phi_{2}$ and $\Phi_{4}$ from (3.10). From (1.6) with $A$ and $B$ as in (2.4-2.5) we see that

$$
\begin{aligned}
\Phi_{2} & =-\partial_{z} \Phi_{1}+\frac{1}{\mu} \Phi_{3} \\
\lambda \Phi_{4} & =\left(4 k^{2} \mu(\lambda+\mu)-\rho \omega^{2}(\lambda+2 \mu)\right) \Phi_{1}-(\lambda+2 \mu) \partial_{z} \Phi_{3} .
\end{aligned}
$$

Hence, upon substituting (3.13) into (3.10), for eigenfunctions $\Phi(\ell)$ we obtain

$$
\langle\Phi(\ell), \Omega\rangle=\int_{0}^{\infty}\left(\rho \omega^{2} \Phi(\ell)_{1} \Omega_{1}+\frac{1}{\mu} \Phi(\ell)_{3} \Omega_{3}-\Omega_{3} \partial_{z} \Phi(\ell)_{1}+\Omega_{1} \partial_{z} \Phi(\ell)_{3}\right) d z .
$$


The first two terms in the integrand (3.14) look sensible in an inner product, but the last two do not. So, in the form (3.14) our 'inner product' is still very strange.

We are left with some uncomfortable facts regarding the eigenfunction expansion (3.11). Not only can we not guarantee that $(\Phi(\ell), \Phi(\ell)) \neq 0$, but we also have no idea about the completeness of the expansion. Furthermore, because of the form of (3.14), if there is completeness for the expansion (3.11), it can only be for the components $\Omega_{1}$ and $\Omega_{3}$. The components $\Omega_{2}$ and $\Omega_{4}$ are subsidiary quantities. Note that the theory of nonselfadjoint eigenvalue problems, including questions of completeness, is still under development. The interested reader should consult Dunford and Schwartz [5]. Finally, note that we have written (3.11) as a sum over discrete eigenvalues, but the mathematical theory does not ensure the existence of as many as one eigenvalue. In addition, there may be a continuous spectrum, and in fact we expect a continuous spectrum in our problem since it occurs for the case of a homogeneous halfspace. One might try to adapt Titchmarsh's [12] theory to deal with these questions.

4. Solution in a homogeneous elastic layer. In this section we determine the solution of the differential equation (1.6) for waves in an elastic slab with constant density and Lamé parameters. In particular, we show that there is a natural separation into upgoing and downgoing waves. Except for the comments on numerical stability at the end of this section, the material presented here is well known. See, for example, Aki and Richards [1, pp. 279-281].

We use the standard method of solution for a linear system of differential equations with constant coefficients, namely, by using the eigenvectors of $A+k^{2} B$ to construct a system in Jordan normal form. It is easy to see that the eigenvalues of $A+k^{2} B$ are $\pm \nu_{\alpha}$ and $\pm \nu_{\beta}$, where

$$
\nu_{\alpha}=\sqrt{k^{2}-\omega^{2} / \alpha^{2}} \quad \text { and } \quad \nu_{\beta}=\sqrt{k^{2}-\omega^{2} / \beta^{2}}
$$

Here, $\alpha$ is the compressional wave speed

$$
\alpha=\sqrt{(\lambda+2 \mu) / \rho}
$$

and $\beta$ the shear wave speed

$$
\beta=\sqrt{\mu / \rho}
$$

In order to make explicit the branch of the square root, when $k<\omega / \alpha$ we define $\nu_{\alpha}$ to be

$$
\nu_{\alpha}=i \sqrt{\frac{\omega^{2}}{\alpha^{2}}-k^{2}}
$$


It follows from a short calculation that the eigenvectors corresponding to the eigenvalues $\pm \nu_{\alpha}$ are

$$
v_{\alpha( \pm)}=\left(1, \pm \nu_{\alpha}, \pm 2 \mu \nu_{\alpha}, 2 k^{2} \mu-\rho \omega^{2}\right)^{T}
$$

where the superscript $T$ indicates the transpose. Furthermore, the eigenvectors corresponding to $\pm \nu_{\beta}$ are

$$
v_{\beta( \pm)}=\left( \pm \nu_{\beta}, k^{2}, 2 k^{2} \mu-\rho \omega^{2}, \pm 2 k^{2} \mu \nu_{\beta}\right)^{T} .
$$

It is clear from (4.3-4.4) that two eigenvectors of $A+k^{2} B$ coalesce if $\nu_{\alpha} \rightarrow 0$ or $\nu_{\beta} \rightarrow 0$, so that those cases require special consideration. For $\nu_{\alpha} \nu_{\beta} \neq 0$, however, the solution to (1.6) on an interval $z_{0} \leq z \leq z_{1}$ may be represented as

$$
\begin{aligned}
\Phi= & C_{1} v_{\alpha(-)} \exp \left\{-\nu_{\alpha}\left(z-z_{0}\right)\right\}+C_{2} v_{\beta(-)} \exp \left\{-\nu_{\beta}\left(z-z_{0}\right)\right\} \\
& +C_{3} v_{\alpha(+)} \exp \left\{-\nu_{\alpha}\left(z_{1}-z\right)\right\}+C_{4} v_{\beta(+)} \exp \left\{-\nu_{\beta}\left(z_{1}-z\right)\right\} .
\end{aligned}
$$

We have chosen this representation because it makes the maximum values of the exponentials be 1 when the eigenvalues are real.

The case of imaginary $\nu_{\alpha}$ or $\nu_{\beta}$. Note that when the Lamé parameters are real and $\nu_{\alpha}$ or $\nu_{\beta}$ is pure imaginary, it is still possible to use a representation equivalent to (4.5) with real functions. In fact, if $\nu_{\beta}$ is imaginary, the terms

$$
C_{2} v_{\beta(-)} \exp \left\{-\nu_{\beta}\left(z-z_{0}\right)\right\}+C_{4} v_{\beta(+)} \exp \left\{-\nu_{\beta}\left(z_{1}-z\right)\right\}
$$

in (4.5) are equivalent to

$$
\tilde{C}_{2} \Re\left(v_{\beta(+)} \exp \left\{\nu_{\beta}\left(z-z_{0}\right)\right\}\right)+\widetilde{C}_{4} \Im\left(v_{\beta(+)} \exp \left\{\nu_{\beta}\left(z-z_{0}\right)\right\}\right)
$$

for appropriate constants $\widetilde{C}_{2}$ and $\widetilde{C}_{4}$. Here, $\Re$ and $\Im$ denote, respectively, the real and imaginary part. That is, for imaginary $\nu_{\beta}$ we may replace the terms in (4.5) involving $C_{2}$ and $C_{4}$ by the terms

$$
\tilde{C}_{2}\left(\begin{array}{c}
-\left|\nu_{\beta}\right| \sin \zeta \\
k^{2} \cos \zeta \\
\left(2 k^{2} \mu-\rho \omega^{2}\right) \cos \zeta \\
-2 k^{2} \mu\left|\nu_{\beta}\right| \sin \zeta
\end{array}\right)+\tilde{C}_{4}\left(\begin{array}{c}
\left|\nu_{\beta}\right| \cos \zeta \\
k^{2} \sin \zeta \\
\left(2 k^{2} \mu-\rho \omega^{2}\right) \sin \zeta \\
2 k^{2} \mu\left|\nu_{\beta}\right| \cos \zeta
\end{array}\right)
$$

with $\zeta=\left|\nu_{\beta}\right|\left(z-z_{0}\right)$. Analogous modifications may be made to (4.5) for imaginary $\nu_{\alpha}$.

The case of vanishing $\nu_{\alpha}$ or $\nu_{\beta}$. In the special case $\nu_{\alpha}=0$ we see from (4.3) that there is a single eigenvector

$$
v_{\alpha}=\left(1,0,0,-\lambda k^{2}\right)^{T}
$$


In order to find the solution of (1.6) when $\nu_{\alpha}=0$, we find a vector $w_{\alpha}$ such that $\left(A+k^{2} B\right) w_{\alpha}=v_{\alpha}$. One possible choice is

$$
w_{\alpha}=(0,1,2 \mu, 0)^{T}
$$

Therefore when $\nu_{\alpha}=0$, the terms in (4.5) containing $\nu_{\alpha}$ are replaced by

$$
C_{1} v_{\alpha}+C_{3}\left(\left(z-z_{0}\right) v_{\alpha}+w_{\alpha}\right)
$$

Remark. For reasons of numerical stability we want to replace the terms in (4.5) involving $\nu_{\alpha}$ by the terms (4.9), not only when $\nu_{\alpha}=0$, but also when $\left|\nu_{\alpha}\right|$ is smaller than some tolerance.

The analogous modification of (4.5) when $\nu_{\beta} \approx 0$ is to use the eigenvector for $\nu_{\beta}=0$

$$
v_{\beta}=\left(0, k^{2}, k^{2} \mu, 0\right)^{T}
$$

together with the generalized eigenvector

$$
w_{\beta}=\left(1,0,0,2 k^{2} \mu\right)^{T} \text {. }
$$

In particular, the terms in (4.5) involving $\nu_{\beta}$ are to be replaced by

$$
C_{2} v_{\beta}+C_{4}\left(\left(z-z_{0}\right) v_{\beta}+w_{\beta}\right)
$$

when $\left|\nu_{\beta}\right|$ is smaller than some prescribed tolerance.

Outgoing boundary conditions. We turn now to the question of the meaning of the outgoing boundary conditions (2.7) for (1.6) and (3.4) for the adjoint equation (1.7). For the case when the slab is a halfspace and the Lamé parameters are real with $\nu_{\alpha}$ and $\nu_{\beta}$ real, it is clear that in order to ensure boundedness of $\Phi$ as $z \rightarrow \infty$, we must take $C_{3}=0$ and $C_{4}=0$. More specifically, we set $C_{3}=0$ in a halfspace whenever $\Re \nu_{\alpha}>0$ and $C_{4}=0$ when $\Re \nu_{\beta}>0$. By (4.9) and (4.10) we also set $C_{3}=0$ when $\nu_{\alpha}=0$ and $C_{4}=0$ when $\nu_{\beta}=0$.

Similar considerations apply to the adjoint equation (1.7). If the slab is a halfspace, we keep only the decaying exponentials.

Consider now the case of pure imaginary $\nu_{\beta}$ for a homogeneous halfspace. Analogous considerations apply to pure imaginary $\nu_{\alpha}$. Then according to the branch $\Im \nu_{\beta}>0$ of the square root defined in (4.2), the factor $\exp \left\{-\nu_{\beta} z\right\}$ in (4.5) corresponds to a downgoing wave, while $\exp \left\{\nu_{\beta} z\right\}$ produces an upgoing wave. The reason for this is contained in the Fourier-Bessel representation (2.2-2.3), since the factor

$$
\exp \left\{i\left(\omega t-\left|\nu_{\beta}\right| z\right)\right\}
$$


gives propagation in the direction of increasing $z$. Hence, for a halfspace we use the representation (4.5) with $C_{3}=0$ and $C_{4}=0$, even when $\nu_{\alpha}$ or $\nu_{\beta}$ is imaginary.

For the adjoint equation (1.7) in a homogeneous halfspace with pure imaginary $\nu_{\beta}$ with $\Im \nu_{\beta}>0$ we again set to zero the coefficient of the exponential $\exp \left\{\nu_{\beta} z\right\}$. The reason for this is that we want (3.2) to hold at $z=\infty$, even though we are only able to impose it in a weak sense. We have already determined that $\Phi$ may contain an exponential of the form $\exp \left\{-i\left|\nu_{\beta}\right| z\right\}$, which represents an outgoing wave. An exponential $\exp \left\{i\left|\nu_{\beta}\right| z\right\}$ in $\Psi$ would therefore cause (3.2) to be violated at $z=\infty$. A term involving $\exp \left\{-i\left|\nu_{\beta}\right| z\right\}$ in $\Psi$, on the other hand, raises the question in (3.2) of the value of the limit

$$
\lim _{z \rightarrow \infty} \exp \left\{-2 i\left|\nu_{\beta}\right| z\right\}
$$

This limit does not exist in the usual sense, but it is zero in the sense of distributions. The meaning of (3.4) for the case of pure imaginary $\nu_{\beta}$ is that $\Psi$ may contain a term involving $\exp \left\{-i\left|\nu_{\beta}\right| z\right\}$ but no term with $\exp \left\{i\left|\nu_{\beta}\right| z\right\}$. Analogous considerations apply to the case of pure imaginary $\nu_{\alpha}$.

5. The problem of a stack of elastic layers. The discussion of the previous section generalizes very easily to the problem of a stack of homogeneous elastic layers. Some care needs to be taken, however, to implement the linear algebra in a manner which is numerically stable. See the papers by Chin, et al. [4] and by Schmidt and Jensen [10]. In each layer we represent the solution of the $P-S V$-system (1.6) in the form given by (4.5), modified as in (4.6), (4.9) or (4.10) if necessary. Thus, there are four unknowns $C_{1}, C_{2}, C_{3}$, and $C_{4}$ associated with each layer. Across each interface between two layers we have the condition that the displacement and stress are continuous. That is, the vector $\Phi$ is continuous across interfaces, and this condition imposes four linear constraints on the $C$ 's for the two layers. In particular, for the representation (4.5) at the interface $z=z_{j}$ we have

$$
\begin{aligned}
& C_{1, j} v_{\alpha(-), j} \exp \left\{-\nu_{\alpha, j}\left(z_{j}-z_{j-1}\right)\right\} \\
& \quad+C_{2, j} v_{\beta(-), j} \exp \left\{-\nu_{\beta, j}\left(z_{j}-z_{j-1}\right)\right\}+C_{3, j} v_{\alpha(+), j}+C_{4, j} v_{\beta(+), j} \\
& =C_{1, j+1} v_{\alpha(-), j+1}+C_{2, j+1} v_{\beta(-), j+1}+C_{3, j+1} v_{\alpha(+), j+1} \exp \left\{-\nu_{\alpha, j+1}\left(z_{j+1}-z_{j}\right)\right\} \\
& \quad+C_{4, j+1} v_{\beta(+), j+1} \exp \left\{-\nu_{\beta, j+1}\left(z_{j+1}-z_{j}\right)\right\} .
\end{aligned}
$$

We must also take account of the boundary conditions at the top and bottom of the stack of layers. If there is a free surface at the top $(z=0)$, then the stress components $\Phi_{3}$ and $\Phi_{4}$ are zero. We have seen in the previous section that the condition for $\Phi$ to have only downgoing waves at infinity is that $C_{3}=0$ and $C_{4}=0$ in (4.5).

In summary, we see that each of these boundary conditions imposes two linear constraints on the coefficients $C_{1}, \ldots, C_{4}$ in (4.5). The boundary conditions and 
the interface conditions (5.1) produce a square matrix equation $M C=0$, where $M$ has the block structure

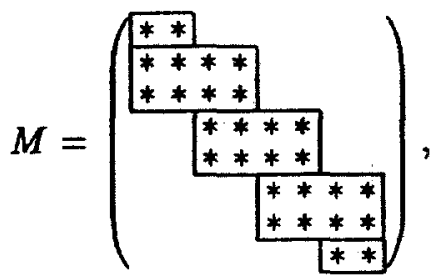

with each asterisk representing a 2-by-2 block. Each pair of columns of the matrix $M$ in (5.2) is associated with the coefficients $C$ in a layer. The first and last block rows of $M$ represent the boundary conditions, and the remaining block rows represent the continuity at interfaces.

We have transformed the problem of finding modes for the stack of layers to one of finding pairs of values $(\omega, k)$ for which $\operatorname{det}(M)=0$, and the corresponding nonzero vector $C$ with $M C=0$ gives the the eigenfunction $\Phi$ via the representation (4.5), with possible modifications (4.6), (4.9), or (4.10). Note that with changing real values of $k$, the transitions from (4.5) to (4.6) to (4.9) are done in such a way as not to introduce any extraneous changes in the sign of $\operatorname{det}(M)$. The computation of $\operatorname{det}(M)$ is based on the matrix factorization associated with Gaussian elimination, since

$$
\operatorname{det}(M)=\operatorname{det}(L) \operatorname{det}(U)
$$

if $M=L U$. One must be very careful about the numerical linear algebra in this factorization, particularly when the matrix $M$ is nearly singular. We also do not want to destroy the block structure of (5.2) by doing Gaussian elimination with unrestricted row pivoting. We therefore use a Gaussian elimination algorithm with row pivoting within blocks. We also modify the Gaussian elimination so as to proceed alternately from the top and bottom of the matrix $M$, choosing the row with the larger pivot. Thus, we do not need to rely on the migration of the nearzero pivot to the last row of the matrix. We have found this strategy to be essential when there are Stoneley waves, since it is our experience that a wave at an interface generally produces a very small pivot element in the block corresponding to that interface.

Real eigenvalues are usually found by holding $\omega$ constant and looking for sign changes in $\operatorname{det}(M)$ as $k$ varies. The reader should be warned that this approach carries a risk of jumping over pairs of very close eigenvalues. This risk is quite real, since it is common for occasional pairs of eigenvalues to come close together. Because this phenomenon depends very strongly on the frequency $\omega$, one ought to solve the eigenvalue problem for many values of $\omega$. The behavior generally observed in the computations is as follows. The eigenvalues vary continuously with $\omega$. For small values of $\omega$ there is always at least one eigenvalue, the one corresponding to a Rayleigh wave. As $\omega$ increases, new (larger) eigenvalues appear, one at a time. 
The user therefore ought to be suspicious of spontaneous creation or disappearance of pairs of eigenvalues as $\omega$ varies. There is, however, no mathematical theory to justify these comments.

Woodhouse [14] has proposed a method, based on the minors of $M$, to be certain of not skipping any eigenvalues. Alternatively, one might consider making checks of the eigenvalue count by using the theorem of Cauchy that for any closed path $\Gamma$ in the complex $k$-plane the value of the integral

$$
I=\frac{1}{2 \pi i} \int_{\Gamma} \frac{\partial_{k} \operatorname{det}(M)}{\operatorname{det}(M)} d k
$$

is the number of zeros of $\operatorname{det}(M)$ inside $\Gamma$. In order to maintain analyticity of $\operatorname{det}(M)$ as a function of $k$, one must be consistent in the representation of the solution, such as by always using the exponential form (4.5). This has the unpleasant side effect of introducing an extraneous zero whenever some layer has $\nu_{\alpha}=0$ or $\nu_{\beta}=0$, since two columns of $M$ then become identical. Another difficulty with the idea using (5.3) is that quadrature and roundoff errors can be very severe, particularly when the path $\Gamma$ passes close to an eigenvalue, (a pole of (5.3)).

When loss is taken into account, the Lamé parameters become complex, so that we can no longer search only for real eigenvalues. There are four common approaches to finding complex zeros: (1) perturbation from the lossless case, (2) Newton's method, (3) counting zeros by the use of (5.3), and (4) making a larger real problem in which $(\Re k, \Im k)$ are zeros of $(\Re \operatorname{det}(M), \Im \operatorname{det}(M))$. Each of these methods has its drawbacks, and more work needs to be done on the development of algorithms for finding zeros of analytic functions in the complex plane.

Computation of the eigenfunctions. In order to find a nontrivial solution of $M C=0$ once we have located an eigenvalue, we use inverse iteration. In this method we compute a sequence of approximations $C(n)$ as follows. We select a nonzero vector $C(0)$ and compute successive iterates

$$
C(n)=s_{n} M^{-1} C(n-1)
$$

where $s_{n}$ is a scale factor to force the entry in the vector $C(n)$ with largest absolute value to be exactly 1 . The iterations (5.4) are easy to carry out because in our computation of $\operatorname{det}(M)$ we already did a factorization $M=L U$ by Gaussian elimination. Once the sequence (5.4) has converged so that $C(n) \approx C(n-1)$, we have the coefficients of an eigenfunction.

6. Fluid layers. We now specialize the equations (1.6), (2.4-2.5) for an elastic layer to the case of a fluid. A fluid differs from a solid in that it has no shear 
strength, so that $\mu=0$. If we set $\mu=0$ in (2.4-2.5), we find that

$$
A=\left(\begin{array}{cccc}
0 & -1 & \infty & 0 \\
0 & 0 & 0 & 1 / \lambda \\
-\rho \omega^{2} & 0 & 0 & -1 \\
0 & -\rho \omega^{2} & 0 & 0
\end{array}\right)
$$

and

$$
B=\left(\begin{array}{llll}
0 & 0 & 0 & 0 \\
1 & 0 & 0 & 0 \\
0 & 0 & 0 & 0 \\
0 & 0 & 1 & 0
\end{array}\right)
$$

Because of the infinity in the third column of $A$ in (6.1), it follows from the definition (2.3) of $\Phi$ that for a fluid we must have $\tau_{r z}=0$ and (for $m \neq 0$ ) $\tau_{z \theta}=0$. That is, a fluid cannot support shear stress. With $\Phi_{3}=0$ and with $A$ and $B$ as in $(6.1-6.2)$ the third row of the system (1.6) gives the identity

$$
\rho \omega^{2} \Phi_{1}=-\Phi_{4}
$$

The variable $\Phi_{1}$ may therefore be eliminated to give the reduced system for a fluid layer, and we obtain the eigenvalue equation (1.6) with

$$
\begin{gathered}
\Phi=\left(\Phi_{2}, \Phi_{4}\right)^{T} \\
A=\left(\begin{array}{cc}
0 & 1 / \lambda \\
-\rho \omega^{2} & 0
\end{array}\right),
\end{gathered}
$$

and

$$
B=\left(\begin{array}{cc}
0 & -1 /\left(\rho \omega^{2}\right) \\
0 & 0
\end{array}\right)
$$

The boundary condition at a free surface is that $\tau_{x z}=0$, so that $\Phi_{4}=0$. We remark that in (6.4) we have chosen to work with the stress in (6.4) because it gives variables which are continuous across a fluid-solid interface. It is more common in underwater acoustics to base the analysis, instead, on the acoustic pressure $p=-\tau_{z z}$.

The representation of (4.3-4.5) in terms of upgoing and downgoing waves extends naturally to the fluid case. The matrix

$$
A+k^{2} B=\left(\begin{array}{cc}
0 & \left(\frac{1}{\lambda}-\frac{k^{2}}{\rho \omega^{2}}\right) \\
-\rho \omega^{2} & 0
\end{array}\right)
$$

has eigenvalues $\pm \nu$ with

$$
\nu=\sqrt{k^{2}-\rho \omega^{2} / \lambda}
$$

That is, the speed of sound in the fluid is given by

$$
\alpha=\sqrt{\lambda / \rho}
$$


The eigenvectors of the matrix in (6.7) are

$$
v_{( \pm)}=\left( \pm \nu,-\rho \omega^{2}\right)^{T}
$$

The fluid analogue of (4.5) is therefore

$$
\Phi=C_{1} v_{(-)} \exp \left\{-\nu\left(z-z_{0}\right)\right\}+C_{2} v_{(+)} \exp \left\{-\nu\left(z_{1}-z\right)\right\}
$$

on the interval $z_{0} \leq z \leq z_{1}$.

The case of imaginary $\nu$. It is clear that in analogy with the case of imaginary $\nu_{\alpha}$ or $\nu_{\beta}$ in the elastic solution (4.5), it is possible to write a real form of $(6.10)$ when $\nu$ in (6.8) is imaginary. Thus we have

$$
\Phi=\tilde{C}_{1}\left(\begin{array}{c}
-|\nu| \sin \zeta \\
-\rho \omega^{2} \cos \zeta
\end{array}\right)+\tilde{C}_{2}\left(\begin{array}{c}
|\nu| \cos \zeta \\
-\rho \omega^{2} \sin \zeta
\end{array}\right)
$$

with $\zeta=|\nu|\left(z-z_{0}\right)$.

The case of vanishing $\nu$. Just as in the elastic case, we see from (6.9) that the eigenvectors $v_{( \pm)}$coalesce when $\nu \rightarrow 0$. Consequently, for $\nu=0$ we use the eigenvector

$$
v=\left(0,-\rho \omega^{2}\right)^{T}
$$

and the generalized eigenvector

$$
w=(1,0)^{T}
$$

such that $\left(A+k^{2} B\right) w=v$. For purposes of numerical stability, whenever $|\nu|$ is smaller than some tolerance, we replace the representation $(6.10)$ by the solution for $\nu=0$,

$$
\Phi=C_{1} v+C_{2}\left(\left(z-z_{0}\right) v+w\right)
$$

with $v$ and $w$ as in (6.11-6.12).

It is clear that for a stack of fluid layers the continuity of the vertical displacement $u_{z}$ and the component of the stress $\tau_{z z}$ produces a matrix equation with structure given by (5.2). The only difference is that the asterisks are 1-by-1 blocks for a fluid.

Conditions at a fluid-solid interface. There remains the question of boundary conditions for a fluid-solid interface. In order to maintain contact, we must have continuity of the vertical displacement $u_{z}$, so that one of the interface conditions is

$$
\Phi_{2}(\text { water })-\Phi_{2}(\text { solid })=0 \text {. }
$$

Similarly, the continuity of the component $\tau_{z z}$ of the stress implies that

$$
\Phi_{4}(\text { water })-\Phi_{4}(\text { solid })=0 \text {. }
$$


The vanishing of the shear components of the stress, $\tau_{r z}$ and $\tau_{z \theta}$, gives the final interface condition

$$
-\Phi_{3}(\text { solid })=0 \text {. }
$$

Note that no condition is imposed on the horizontal displacement $\Phi_{1}$ (solid) at a fluid-solid interface.

The adjoint equation for a fluid. With $\Phi$ as in (6.4) and with $A$ and $B$ as given by (6.5-6.6), we see from direct substitution that

$$
\Psi=\left(\begin{array}{c}
-\Phi_{4} \\
\Phi_{2}
\end{array}\right)
$$

is a solution of the adjoint equation (1.7). Furthermore, we see that

$$
\Psi(\ell)^{T} B \Omega=\frac{\Phi(\ell)_{4} \Omega_{4}}{\rho \omega^{2}}
$$

which gives the standard weighted inner product in acoustics. Observe also, that (6.21) is the integrand of (3.14) under conditions appropriate to a fiuid, namely, the vanishing of the shear components $\Phi(\ell)_{3}$ and $\Omega_{3}$. Thus, in computing inner products' we use (3.10) or (3.14) over elastic layers and integrate (6.21) over fluid layers.

Aknowledgement. This work was supported by the High Gain Initiative under Dr. R. Doolittle of the Office of Naval Research.

\section{References}

[1] K. Aki and P. G. Richards, Quantative Seismology, Freeman, San Freancisco, 1980.

[2] J. I. Arvelo, M. Talmant, and H. Überall, "A normal-mode model of underwater propagation including shear effects in a layered ocean floor", pp. 131-150 in Computational Acoutsics, vol. 3, D. Lee, A. Cakmak, and R. Vichnevsky, eds., Elsevier, Amsterdam, 1990.

[3] C. H. Chapman and J. H. Woodhouse, "Symmetry of the wave equation and excitation of body waves', Geophys. J. R. astr. Soc. 65 (1981), 777-782.

[4] R. C. Y. Chin, G. W. Hedstrom, and L. Thigpen, "Matrix methods in synthetic seismograms", Geophys. J. R. astr. Soc. 77 (1984), 483-502.

[5] N. Dunford and J. T. Schwartz, Linear Operators, vol. 3, Wiley-Interscience, New York, 1971.

[6] A. C. Eringen Mechanics of Continua, Krieger, Malabar, Florida, 1980. 
[7] B. L. N. Kennett, Seismic Wave Propagation in Stratified Media, Cambridge University Press, 1983.

[8] M. B. Porter and E. L. Reiss, "A numerical method for bottom interacting ocean acoustic normal modes", J. Acoust. Soc. Am. 77 (1985), 1760-1767.

[9] S. A. Preuss, "Solving linear boundary value problems by appsoximating the coefficients", Math. Comput. 27 (1973), 551-561.

[10] H. Schmidt and G. Tango, "Efficient global matrix approach to the computation of synthetic seismograms", Geophys. J. R. astr. Soc. 84 (1986), 331-359.

[11] H. Takeuchi and M. Saito, "Seismic surface waves", pp. 217-295 in Methods of Computational Physics, vol. 11, B. A. Bolt, ed., Academic Press, New York, 1972.

[12] E. C. Titchmarsh, Eigenfunction Expansions, vol. 1, Oxford University Press, 1946.

[13] J. H. Woodhouse, "Asymptotic results for elastodynamic propagator matrices in plane stratified and spherically stratified earth models", Geophys. J. R. astr. Soc. 54 (1978), 263-280.

[14] J. H. Woodhouse, "The calculation of eigenfrequencies and eigenfunctions of the free oscillations of the earth and the sun", pp. 321-370 in Seismological Algorithms, D. J. Doornbos, ed., Academic Press, 1988. 


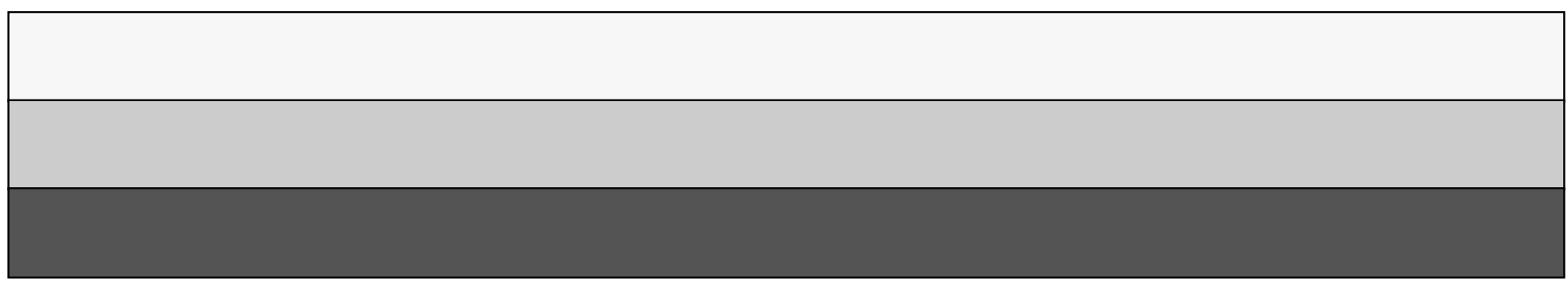

